\title{
Epidemiología de la infección oral por VPH en sujetos jóvenes sanos
}

\author{
Beddler G. Cab-Sánchez, Sandra E. Hernández-Solís, Florencio Rueda-Gordillo, Laura Conde-Ferráez, \\ Jesús G. Gómez-Carballo y María del Refugio González-Losa
}

\section{Epidemiology of oral HPV infection in young healthy individuals}

Background: HPV infection is the most common sexually transmitted viral infection, and is associated with several neoplasms. Aim: To describe the epidemiology, natural history and risk factors associated with oral HPV infection in asymptomatic young adults. Methods: A prospective and longitudinal study was conducted, including subjects without oral pathology, who were sampled from the oral mucosa. All subjects with positive results were re-sampled 6 months later. The presence of HPV was identified by PCR. Demographic and sexual behavior data were obtained with a survey that was responded without the intervention of the researcher. Results: 102 samples were collected from subject of 18-26 years old, 60 (58.8\%) were male. The prevalence of the virus was $6.9 \%$; all positive subjects had active sexual life. Same-gender relationships were the only variable associated with the presence of the virus $(\mathrm{p}<0.05)$. At six months all subjects had eliminated the virus. Conclusion: Oral HPV infection is transient and is associated to same-gender relationships, mainly women who have sex with women.

Key words: Oral papillomavirus, epidemiology, Mexico, young, HPV.

Palabras clave: Papilomavirus, oral, epidemiologia, México, joven, VPH.

\section{Introducción}

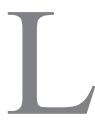
a infección por virus de papiloma humano (VPH) es la infección viral de transmisión sexual más frecuente, presente en todos los continentes y razas, con impacto en la morbilidad y mortalidad mundial. Los VPH pertenecen a la familia de Papillomaviridae, son virus no envueltos, de ADN de doble cadena, con aproximadamente 8.000 pares de bases, que se encuentran ampliamente distribuidos en la naturaleza y presentes en diversas especies. Los VPH infectan el epitelio escamoso estratificado de piel y mucosas, ocasionando lesiones proliferativas benignas y malignas ${ }^{1}$.

A la fecha, se ha identificado más de un centenar de tipos VPH, los cuales se han clasificado en virus no oncogénicos y oncogénicos por su capacidad de inducir transformación maligna ${ }^{2}$. El potencial oncogénico de los VPH ha sido establecido por décadas en estudios biológicos y epidemiológicos; en 2012, fueron responsables de 640.000 casos nuevos de neoplasias ${ }^{3}$.

Las neoplasias de cabeza y cuello son un grupo heterogéneo de cánceres que se originan en el tracto aéreo y digestivo superior; en una fracción de ellos se ha encontrado el genoma del VPH con frecuencias que varían de acuerdo a la región anatómica. La mayor frecuencia se reporta en las neoplasias que se originan en orofaringe, con una frecuencia mayor a $20 \%{ }^{4}$. Lesiones benignas (verrugas, condilomas, o hiperplasia epitelial multifocal) causadas por el VPH se presentan en la cavidad oral de sujetos de todas las edades ${ }^{5,6}$, de manera que estos microorganismos son importantes agentes etiológicos de patología oral.

La presencia del genoma viral en la cavidad oral de sujetos sin patología ha sido analizada por diversos autores con frecuencias que varían de 0,6 a $81 \% \%^{7-10}$. Se ha asociado al sexo oral sin protección como el principal factor de riesgo a la infección oral por genotipos presentes en el tracto genito-urinario ${ }^{11,12}$. La evolución natural de la infección oral por VPH ha sido poco estudiada en sujetos inmunocompetentes ${ }^{13,14}$. El objetivo de este estudio es contribuir al conocimiento de la epidemiología, historia natural y factores de riesgo asociados a la infección oral por VPH en adultos jóvenes sin lesiones.

\section{Material y Métodos}

\section{Población de estudio}

Se realizó un estudio prospectivo, longitudinal, observacional y analítico, que incluyó 102 sujetos de 18 a 26 años de edad, que acudieron voluntariamente para consulta odontológica de rutina a la Unidad de Atención Integral de la Salud (UAIS) de la Universidad Autónoma de Yucatán (UADY), en Mérida, Yucatán, México. Se incluyeron pacientes de uno u otro sexo, sin patología de la mucosa oral; los sujetos con antecedentes de lesiones
Universidad Autónoma de Yucatán. Mérida, Yucatán, México.

Facultad de Odontología Departamento de Microbiología Oral y Biología Molecular (BGCS, SEHS, FRG).

Centro de Investigaciones Regionales "Dr. Hideyo Noguchi" Campus de Ciencias de la Salud Unidad de Ciencias Biomédicas Inalámbrica (LCF, JGGC, MRGL)

Los autores declaran que no existen conflictos de interés Financiamiento: Universidad Autónoma de Yucatán.

Recibido: 6 de julio de 2017 Aceptado: 24 de octubre de 2017

Correspondencia a: María del Refugio González-Losa glosa@correo.uady.mx 
orales por VPH fueron eliminados. Previamente a la recolección de datos y toma de muestra se proporcionó información clara y concisa sobre los objetivos y procedimientos del proyecto; los sujetos que aceptaron participar firmaron una carta de consentimiento informado.

\section{Recolección de datos y muestras}

Se recolectaron muestras de mucosa de pilares amigdalinos, amígdalas, paladar, carrillos, lengua, piso de boca y labios con hisopo de dacrón, que se depositó en un tubo con $2 \mathrm{ml}$ de etanol al 50\% como medio de transporte y se almacenó a temperatura ambiente hasta su análisis. Todos los pacientes que resultaron positivos fueron informados de manera personal y se procedió a recolectar una segunda muestra con el mismo procedimiento seis meses después de la primera toma. Las muestras fueron procesadas en el laboratorio de Virología del Centro de Investigaciones Regionales "Dr. Hideyo Noguchi” de la UADY. Con posterioridad a la toma de muestra, se les proporcionó una encuesta que fue contestada de manera personal y privada sin necesidad de intervención del investigador para conocer variables epidemiológicas: sexo, edad, lugar de residencia, estado civil, escolaridad, ocupación. Variables de conducta sexual: edad de inicio de vida sexual activa, preferencia sexual, número de parejas sexuales, practica de sexo oral, uso de condón, antecedentes de verrugas genitales en el paciente o algún compañero, tabaquismo y antecedentes de enfermedades crónicas.

\section{Detección y genotipos de $\mathrm{VPH}$}

La extracción del material genético se realizó de manera automatizada utilizando el MagNa Pure LC Total Nucleic Acid Isolation Kit (Roche). Para evaluar la calidad del ADN, se amplificó una región de $240 \mathrm{pb}$ del gen de la $\beta$-globina humana, utilizando metodología previamente descrita ${ }^{15}$. La determinación del virus se realizó por medio de la amplificación génica de un segmento de 450 pb del gen L1 (que es una región conservada en todos los genotipos que infectan mucosas) utilizando los oligonucleótidos llamados "universales" MY09/MY1116. Todos los amplificados se visualizaron en geles de acrilamida al $8 \%$, teñidos con nitrato de plata $1 \mathrm{M}$, utilizando un marcador de $100 \mathrm{pb}$ (Invitrogen).

Para la identificación de los genotipos se utilizó una reacción de polimerasa en cadena (RPC) anidada, múltiple, que amplifica un segmento de los oncogenes E6 y E7 de tamaño variable de acuerdo al genotipo amplificado, siguiendo metodología descrita previamente ${ }^{17}$. En el Estado de Yucatán, México, la infección por VPH 13 es común ${ }^{6}$, por lo que las muestras negativas fueron procesadas en busca de este genotipo amplificando un segmento de 240 pb del gen L1 de acuerdo a la metodología reportada por Williamson y Dennis ${ }^{18}$.

\section{Análisis estadístico}

Las variables analizadas fueron: vida sexual, género, edad de inicio de vida sexual, número de compañeros sexuales, tipo de relaciones sexuales, sexo oral, uso de preservativos durante relaciones sexuales no orales y orales, consumo de alcohol y tabaquismo. La edad de inicio de vida sexual se categorizó en $\leq 18$ años de edad vs $\geq 19$ años; en el caso de tipo de relaciones sexuales se analizaron relaciones con personas del mismo sexo vs relaciones sexuales con personas solo de otro sexo $\mathrm{y}$ ambos sexos. El número de compañeros sexuales fue dividido en tres grupos: $<5,5-10 \mathrm{y}>10$. En el caso de uso de condón durante cualquier relación sexual o específicamente sexo oral, las opciones de respuesta eran siempre, nunca y ocasionalmente, y sólo se examinó como opción independiente el sexo oral; no se hizo distinción entre anal y vaginal. Para determinar la asociación de las variables y la presencia de VPH, se realizaron con la prueba estadística de $\chi^{2}$, con un nivel de confianza del $95 \%$, considerando como asociación toda $\mathrm{p}=<0,05$. El análisis se realizó en el programa Statgraphics plus 5.1.

\section{Resultados}

\section{Características de la población}

Se recolectaron 102 muestras de sujetos de 18-26 años de edad, con una media de 22 años; 60 (58,8\%) correspondieron al sexo masculino.

El 83,3\% (85/102) había iniciado vida sexual al momento del estudio; $23,8 \%(10 / 42)$ y $11,7 \%$ (7/60) de las mujeres y hombres, respectivamente, negaron vida sexual.

El promedio de la edad de inicio de vida sexual fue 17,3 años (14-25 años); 54,1\% (46/85) había iniciado vida sexual $\leq 17$ años. El promedio de parejas sexuales fue de 3,8 y $73,8 \%$ (62/84) refirieron haber tenido cuatro parejas o menos, $20,2 \%$ (17/84) entre 5 y 9 y $5,9 \%$ (5/84) más de 10 parejas sexuales. Uno de los sujetos con vida sexual reportó que desconocía el dato exacto.

Respecto al tipo de relaciones sexuales que refirieron los participantes, $75,3 \%$ fueron heterosexuales; $20,0 \%$ homosexuales y $4,7 \%$ bisexuales. El sexo oral es una práctica común en 77,6\% (66/85) de los jóvenes. El 10,6\% (7/66) dijeron utilizar siempre condón durante esta práctica; $15,2 \%(10 / 66)$ ocasionalmente y 74,2\% (49/66) nunca.

El 22,5\% (23/102) eran fumadores; 32,4\% (33/102) estuvieron expuestos al humo de tabaco al convivir con fumadores y 58,4\% (59/101) afirmaron consumir alcohol; un encuestado no tenía respuesta a esta pregunta.

\section{Prevalencia y genotipos de $\mathrm{VPH}$}

Al analizar la calidad del ADN de las muestras de estudio, $100 \%$ amplificó para el gen de $\beta$-globina humana. La prevalencia global de VPH fue de 6,9\% (7/102); 11,9\% $(5 / 42)$ de las mujeres y $3,3 \%(2 / 60)$ de los hombres fueron 
positivos. Todas las muestras positivas eran de jóvenes sexualmente activos; de manera que la prevalencia en esta población fue de $8,2 \%(7 / 85)$; siendo mayor en las mujeres que en los hombres $15,6 \%(5 / 32)$ y $3,7(2 / 53)$, respectivamente.

Respecto a los genotipos, se encontró que el más frecuente fue VPH 13 en tres muestras; VPH 45, VPH 6/11 y VPH 18 se encontraron en una muestra cada uno. Una de las muestras no pudo ser genotipificada ya que no amplificó con ninguno de los iniciadores específicos de genotipos. Tomando en cuenta sólo la población sexualmente activa, podemos decir que 2,4\% (2/85) tenía un genotipo de alto riesgo, y 4,7\% (4/85) de bajo riesgo. Ahora bien, con respecto a los sujetos positivos, $28,6 \%$ $(2 / 7)$ era portador de un genotipo oncogénico. A los siete sujetos se les tomó una segunda muestra a los seis meses $\mathrm{y}$ todos resultaron negativos al virus.

\section{Características y conductas asociadas a la infección oral por $\mathrm{VPH}$}

Todos los sujetos positivos ya tenían vida sexual activa; el promedio de edad de los siete sujetos positivos era 21,3 años; dos son varones y cinco mujeres. La edad promedio de inicio de vida sexual activa fue de 18,7 años; el promedio de vida sexual al momento del estudio era de 2,5 años, seis jóvenes reportaron $\leq$ tres años de vida sexual. Cinco jóvenes dijeron haber tenido dos parejas sexuales, y dos sólo una. Solamente una mujer negó sexo oral.

Respecto a la utilización de condón durante el sexo oral dos refirieron uso ocasional, y cinco lo negaron. Respecto al tipo de relaciones sexuales, tres mujeres y un hombre refirieron relaciones con el mismo sexo; dos mujeres refirieron tener relaciones con varones y un varón con ambos sexos. Todos eran consumidores de alcohol, aunque no se cuantificó y tres eran fumadores y una, fumadora pasiva.

Para analizar si la vida sexual activa está asociada a la presencia del virus, se incluyó toda la población estudiada, pero para el análisis de las otras variables solamente se incluyó a la población sexualmente activa (n: 85), debido a que sólo en este grupo se encontró al virus. A pesar de que sólo sujetos con vida sexual fueron positivos al virus, al hacer el análisis estadístico no se encontró asociación (p: 0,456). De todas las variables estudiadas, tener relaciones sexuales con personas del mismo sexo fue la única variable asociada la presencia de ADN de VPH en cavidad oral (p: 0,0383) (Tabla 1).

\section{Discusión}

En este estudio, se analizó la presencia de ADN de VPH en células de la mucosa oral de un grupo de jóvenes sin patología oral, ni antecedentes de lesiones causadas por el virus. La prevalencia global de VPH fue de 6,9\%
Tabla 1. Conducta sexual y su relación con la infección oral por VPH en una cohorte de 85 sujetos sanos

\begin{tabular}{|c|c|c|c|}
\hline Variable & Positivo a VPH & Negativo a VPH & Valor de $p$ \\
\hline \multicolumn{4}{|l|}{ Género } \\
\hline Masculino & 2 & 51 & \multirow[b]{2}{*}{0,1289} \\
\hline Femenino & 5 & 27 & \\
\hline \multicolumn{4}{|c|}{ Edad inicio de vida sexual } \\
\hline$\leq 18$ años & 1 & 45 & \multirow[b]{2}{*}{0,0700} \\
\hline$\geq 19$ años & 6 & 33 & \\
\hline \multicolumn{4}{|c|}{ Número de parejas sexuales } \\
\hline$<5$ & 7 & 55 & \multirow{3}{*}{0,243} \\
\hline $5-10$ & 0 & 8 & \\
\hline$>10$ & 0 & 15 & \\
\hline \multicolumn{4}{|l|}{ Sexo oral } \\
\hline Sí & 6 & 60 & \multirow[b]{2}{*}{0,951} \\
\hline No & 1 & 18 & \\
\hline \multicolumn{4}{|c|}{ Uso condón en relaciones no orales } \\
\hline Siempre & 1 & 45 & \multirow{3}{*}{0,128} \\
\hline Ocasionalmente & 3 & 23 & \\
\hline Nunca & 2 & 10 & \\
\hline \multicolumn{4}{|c|}{ Uso de condón en sexo orala } \\
\hline Siempre & 0 & 7 & \multirow{3}{*}{0,492} \\
\hline Ocasionalmente & 2 & 10 & \\
\hline Nunca & 5 & 48 & \\
\hline \multicolumn{4}{|c|}{ Relaciones sexuales con mismo sexo } \\
\hline Sí & 4 & 13 & \multirow[b]{2}{*}{$0,0383^{*}$} \\
\hline No & 3 & 65 & \\
\hline \multicolumn{4}{|l|}{ Tabaquismo } \\
\hline Sí & 3 & 19 & \multirow[b]{2}{*}{0,535} \\
\hline No & 4 & 59 & \\
\hline \multicolumn{4}{|l|}{ Consumo de alcohol } \\
\hline Sí & 7 & 47 & \multirow[b]{2}{*}{0,099} \\
\hline No & 0 & 30 & \\
\hline
\end{tabular}

*Diferencia estadísticamente significativa. ${ }^{\circledR} 13$ sujetos no dieron esa información.

(7/102): $11,9 \%$ en mujeres y 3,3\% en varones. Todas las muestras positivas eran de jóvenes sexualmente activos; de manera que, si eliminamos a los participantes que no tenían vida sexual activa, se incrementa a 8,2\% (7/85); siendo mayor en las mujeres que en los hombres 15,6 y $3,8 \%$, respectivamente.

Recientemente, un estudio realizado de 2009 a 2012 analizó la presencia del virus en la cavidad oral de 9,257 sujetos de ambos sexos, de 18 a 65 años de edad y de diversos orígenes étnicos, todos residentes de los Estados Unidos de América (E.U.A.), se encontró una prevalencia de $7,4 \%{ }^{19}$. Kreimer y cols., analizaron 1.688 muestras de varones residentes de Brasil, México y E.U.A., reportando una prevalencia global de $4 \%$; el análisis por país demos- 
tró la mayor prevalencia $(5,9 \%)$ en mexicanos ${ }^{20}$. Otros estudios han documentado prevalencias semejantes a las encontradas en este estudio: Italia 5,5\% ${ }^{21}$; Brasil $6,5 \%{ }^{22}$ y E.U.A. $6,8 \%{ }^{23}$.

En el grupo estudiado, la relación hombre:mujer fue de 1,4:1; sin embargo, la infección fue mayor entre las mujeres que en los varones $11,9 \%$ (5/62) y 3,3\% (2/60), respectivamente; si se comparan sólo los individuos con vida sexual, la diferencia se hace más evidente $15,6 \%$ $(5 / 32)$ para mujeres y $3,8 \%(2 / 53)$ para varones, aunque no hubo una diferencia estadísticamente significativa $(\mathrm{p}>0,05)$. Estudios previos han demostrado una prevalencia mayor en varones ${ }^{8,11,19}$.

Del total de sujetos analizados, el $2 \%$ estaba infectado con un genotipo de alto riesgo (18 y 45), 2,4\% de los que tienen vida sexual y $28,6 \%$ de los positivos al virus. Los genotipos no oncogénicos encontrados fueron: el 13 y $6 / 11$ en $3,9 \%$ de toda la población y $57,1 \%$ de los positivos. Recientemente, un meta-análisis que incluyó 29 estudios, encontró una prevalencia de 2,7 y 2,2\% para genotipos de alto y bajo riesgo, respectivamente ${ }^{11}$. En este estudio, la prevalencia de los genotipos de bajo riesgo fue ligeramente mayor, lo cual puede ser explicada por la presencia del genotipo 13. No se encontraron infecciones dobles en sujeto positivo alguno.

El VPH 13 es un genotipo específico de mucosa oral que ocasiona una patología benigna conocida como hiperplasia epitelial multifocal o enfermedad de Heck descrita en grupos étnicos, principalmente en América. Nuestro grupo ha documentado la presencia de esta patología en comunidades Mayas del sureste de México y de infección asintomática en mujeres en edad reproductiva ${ }^{6,24}$. El 2,9\% de la población estudiada tenía una infección asintomática y sin antecedentes de la patología, reforzando la evidencia epidemiológica de que este genotipo es endémico en el estado de Yucatán, México. Cuberos y cols, en un estudio de casos y controles, reportaron que $29,6 \%$ de los controles eran positivos al virus, siendo éste el único estudio además de los nuestros que han documentado la infección asintomática por este genotipo ${ }^{25}$.

En el presente estudio, el virus se encontró solamente en sujetos con vida sexual; sin embargo, no hubo una asociación estadísticamente significativa ( $\mathrm{p}>0,05)$. Resultado similar fue reportado por Antonsson y cols. quienes analizaron una población semejante (18-35 años de edad $)^{26}$.

La conducta sexual, el tabaquismo y consumo de alcohol han sido implicados como factores de riesgo para la infección por VPH. El número de compañeros sexuales es la conducta sexual que más frecuentemente se ha encontrado asociada a la presencia oral del virus, en una relación directamente proporcional ${ }^{8,11,12,23}$. En la población estudiada, el número de compañeros sexuales totales, el sexo oral, el uso de condón durante relaciones sexuales de cualquier tipo y durante sexo oral específicamente no se asociaron a la presencia del virus. Respecto al sexo oral, no se hizo una diferencia entre recibir o dar sexo oral, diferencia que han analizado otros autores encontrando que recibir sexo oral se asocia a la infección oral por el virus $(p=0,0004)$, no así dar sexo oral ${ }^{26}$. En estudios posteriores es fundamental analizar de una manera independiente la práctica de sexo oral activo y pasivo, para tener evidencias científicas más claras sobre el riesgo del sexo oral activo y el pasivo.

De todas las variables de conducta sexual analizadas, solamente tener relaciones con el mismo sexo se asoció a la presencia del virus $(\mathrm{p}=0,0383)$. El 57,1\% (4/7) de las muestras positivas eran de jóvenes que tenían relaciones sexuales exclusivamente con personas de su mismo sexo; tres de ellas eran mujeres. El hallazgo de la presencia de mujeres que tienen exclusivamente sexo con mujeres al momento del estudio y que se encuentran infectadas siendo esta variable un factor asociado a la infección oral por VPH es relevante a pesar del tamaño de la muestra. Hasta donde es nuestro conocimiento, es la primera ocasión que se reporta que las relaciones sexuales entre mujeres es un factor de riesgo para la infección oral por VPH. Shigeishi y Sugiyama ${ }^{11}$, en un estudio de meta-análisis reportaron una prevalencia superior en los hombres que tienen sexo con hombre (HSH) comparado con mujeres y hombres que tienen sexo con mujeres $(12,2 \%, 2,9 \%$ y $4,7 \%)$.

A pesar de los cambios y apertura a las diferentes expresiones y preferencias sexuales, las mujeres que tienen sexo con mujeres (MSM) es un grupo poco reconocido, mejor dicho, su realidad y necesidades son invisibles, no son contempladas en los programas de salud, a diferencia de lo que ha sucedido con los hombres que tienen sexo con hombres (HSH) que son objeto de campañas de salud, programas de prevención y un sinnúmero de acciones para prevenir patologías propias de su actividad sexual. Incluso, en el ámbito académico, las MSM han sido olvidadas; es hasta la última década que se ha empezado a publicar artículos que van delineando el sentir de este grupo respecto a su riesgo de contraer infecciones de transmisión sexual (ITS), y su participación en los programas de prevención. Las evidencias actuales demuestran que se sienten en menor riesgo de contraer ITS que las mujeres heterosexuales ${ }^{27,28}$, y que sí pueden infectarse con el VPH, aunque nunca hayan tenido una relación heterosexual ${ }^{29,30}$.

Hasta donde es nuestro conocimiento, son pocos los estudios longitudinales para conocer la evolución de la infección por VPH en la cavidad oral de sujetos sin VIH y en mujeres sin patología cervical concomitante. En este estudio, los siete sujetos positivos eliminaron al virus espontáneamente, Debido a que el estudio fue para establecer prevalencia y no incidencia del virus, no se puede establecer el tiempo de eliminación, ya que se desconoce la fecha de inicio de la infección. Estudios 
recientes realizados en hombres, han puesto de manifiesto que más de $50 \%$ de las infecciones son eliminadas antes de los 12 meses; observándose diferencias entre los genotipos $^{31,32}$. Kreimer (2013) analizó una cohorte de 1.626 varones de 18 a 73 años, sin antecedentes de infección de transmisión sexual alguna, que fueron seguidos cada 6 meses por 12,7 meses en promedio. En ellos, 55\% de las infecciones incidentes por cualquier genotipo fueron eliminadas en $6,9(6,2-9,3)$ meses, con diferencias en el tiempo de eliminación entre los genotipos ${ }^{31}$. La presencia de VIH no modifica la evolución natural de la infección oral por VPH, de acuerdo a lo reportado por van Aar y cols., (2014) que efectuaron seguimiento por 12 meses a 433 y 290 sujetos sin y con infección por VIH, respectivamente ${ }^{32}$.

En 15,3\% (111/723) de los sujetos se encontró VPH en la cavidad oral y $53,2 \%$ lo eliminaron en un período de 12 meses. En el análisis multivariado, los autores no encontraron asociación entre la infección por VIH y la eliminación del VPH ( $p=0,167$; IC 95\% 0,2-1,3); la carga viral de VIH y el recuento de linfocitos no se analizaron como posibles factores involucrados en la evolución de la infección oral ${ }^{32}$.

La evolución natural de la infección en el cérvix uterino está ampliamente documentada, sin dejar duda que casi $80 \%$ de las infecciones se eliminan espontáneamente en un período máximo de 18-24 meses ${ }^{1}$. Nuestros resultados, en conjunto con lo descrito por otros autores, ponen de manifiesto que la evolución de la infección es similar en la mucosa oral y cervical; sin embargo, es necesario realizar estudios con cohortes mayores.

Es importantes considerar las limitaciones del estudio: el número de sujetos incluidos fue bajo, y el instrumento de recolección debe ser más detallado. El número y sexo de compañeros sexuales totales y en los últimos seis meses, de acuerdo al tipo de relaciones (orales, cervicales, y/o anales), tipo de sexo oral (activo, pasivo o ambos), cuantificación del consumo de alcohol y tabaco, son algunos de los cambios que deben realizarse en las próximas investigaciones.
Finalmente, los resultados del estudio contribuyen al conocimiento de la epidemiología de la infección oral por VPH en un grupo poco estudiado (jóvenes de ambos sexos, sin VIH), y una de las principales aportaciones es el hecho de hacer visible a un grupo de riesgo ignorado, las mujeres que tienen sexo con mujeres, que requieren ser incluidas en protocolos de investigación para generar información que guíe las políticas de salud que atiendan a sus necesidades específicas.

Paralelamente, evaluar si los jóvenes consideran que el sexo oral es una práctica de riesgo, daría elementos para diseñar campañas de educación sexual basadas en la realidad de la población blanco.

\section{Resumen}

Introducción: La infección por VPH es la infección viral de transmisión sexual más frecuente, y se encuentra asociada a diversas neoplasias. Objetivo: Describir la epidemiología, historia natural y factores de riesgo asociados a la infección oral por VPH en adultos jóvenes asintomáticos. Métodos: Se realizó un estudio prospectivo de corte longitudinal, que incluyó sujetos sin patología oral, a los que se les tomó una muestra de la mucosa bucal. A todos los sujetos con resultados positivos se les realizó un nuevo muestreo seis meses después. Se identificó la presencia del virus por RPC; los datos demográficos y de conducta sexual fueron obtenidos con una encuesta que respondieron sin intervención del investigador. Resultados: Se recolectaron 102 muestras de sujetos de 18-26 años de edad, 60 (58,8\%) correspondieron al sexo masculino. La prevalencia del virus fue de $6,9 \%$; todos los sujetos positivos tenían vida sexual. Las relaciones sexuales entre personas del mismo sexo fue la única variable asociada a la presencia del virus $(\mathrm{p}<0,05)$. A los seis meses, todos los sujetos habían eliminado al virus. Conclusión: La infección oral por VPH es transitoria y está asociada a relaciones sexuales entre personas del mismo sexo, principalmente mujeres que tienen sexo con mujeres.

\section{Referencias bibliográficas}

1.- López-Saavedra A, Lizano-Soberón M. Cáncer cervicouterino y el virus del papiloma humano: la historia que no termina. Cancerología 2006; 1: 31-55.

2.- Bernard H U, Burk R D, Chen Z, van Doorslaer K, zur Hausen H, de Villiers EM. Classification of papillomaviruses (PVs) based on $189 \mathrm{PV}$ types and proposal of taxonomic amendments. Virology 2010; 401: 70-9.

3.- Plummer M, de Martel C, Vignat J, Ferlay J, Bray F, Franceschi S. Global burden of cancers attributable to infections in 2012: a synthetic analysis. Lancet Glob Health 2016; 4 : e609-16.

4.- Prigge E S, von Knebel Doeberitz M, Reuschenbach M. Clinical relevance and implications of HPV-induced neoplasia in different anatomical locations. Mutat Res 2017; 772: 51-66.

5.- Medina M L, Medina M G, Merino L A. Consideraciones actuales sobre la presencia de papilomavirus humano en la cavidad oral. Av Odontoestomatol 2010; 26: 71-80.

6.- López-Villanueva M E, Conde-Ferráez L,
Ayora-Talavera G, Cerón-Espinosa J D, González-Losa M del R. Human papillomavirus 13 in a Mexican Mayan community with multifocal epithelial hyperplasia: could saliva be involved in household transmission? Eur J Dermatol 2011; 21: 396-400.

7.- Kurose K, Terai M, Soedarsono N, Rabello D, Nakajima Y, Burk R D, et al. Low prevalence of HPV infection and its natural history in normal oral mucosa among volunteers on Miyako Island, Japan. Oral Surg Oral Med Oral Pathol Oral Radiol Endod 2004; 98: 91-6.

8.- Gillison M L, Broutian T, Pickard R K, Tong 
Z Y, Xiao W, Kahle L, et al. Prevalence of oral HPV infection in the United States, 2009-2010. JAMA 2012; 307: 693-703.

9.- Colon-López V, Quiñones-Ávila V, Del ToroMejías L M, Reyes K, Rivera M E, Nieves K, et al. Oral HPV infection in a clinic-based sample of Hispanic men. BMC Oral Health 2014; $14: 7$

10.- Pickard R K, Xiao W, Broutian T R, He X, Gillison M L. The prevalence and incidence of oral human papillomavirus infection among young men and women, aged $18-30$ years. Sex Transm Dis 2012; 39: 559-66.

11.- Shigeishi H, Sugiyama M. Risk factors for oral human papillomavirus infection in healthy individuals: a systematic review and metaanalysis. J Clin Med Res 2016; 8: 721-9.

12.- Read T R, Hocking J S, Vodstrcil LA, Tabrizi S N, McCullough M J, Grulich A E, et al. Oral human papillomavirus in men having sex with men: risk-factors and sampling. PLoS One 2012; 7: e49324.

13.- D'Souza G, Fakhry C, Sugar E A, Seaberg E C, Weber K, Minkoff H L, et al. Six-month natural history of oral versus cervical human papillomavirus infection. Int. J Cancer 2007; 121: $143-50$.

14.- Louvanto K, Rautava J, Willberg J, Wideman L, Syrjänen K, Grénman S, et al. Genotypespecific incidence and clearance of human papillomavirus in oral mucosa of women: a six-year follow-up study. PLoS One 2013; 8: e53413.

15.- Saiki R K, Bugawan T L, Horn G T, Mullis $\mathrm{K} \mathrm{B}$, Erlich H A. Analysis of enzymatically amplified beta-globin and HLA-DQ alpha DNA with allele-specific oligonucleotide probes. Nature 1986; 324: 163-6.

16.- Manos M M, Ting Y, Wright D K, Lewis J, Broker T R, Wolinsky S M. The use of polymerase chain reaction amplification for the detection of genital human papillomaviruses. Cancer Cells 1989; 7: 209-14.

17.- Sotlar K, Diemer D, Dethleffs A, Hack Y, Stubner A, Vollmer N, et al. Detection and typing of human papillomavirus by E6 nested multiplex PCR. J Clin Microbiol 2004; 42: 3176-84.

18.- Williamson A L, Dennis S J. The use of the polymerase chain reaction for the detection of human papillomavirus type 13. J Virol Methods 1991; 31: 57-65.

19.- Bui T C, Tran L T, Thai T N, Shete S S, Vidrine D J, Sturgis E M. Prevalence of and risk factors for oral human papillomavirus infection with multiple genotypes in the United States. Sex Transm Dis 2017; 44: 166-72.

20.- Kreimer A R, Villa A, Nyitray A G, Abrahamsen M, Papenfuss M, Smith D, et al. The epidemiology of oral HPV infection among a multinational sample of healthy men. Cancer Epidemiol Biomarkers Prev 2011; 20: 172-82.

21.- Giovannelli L, Campisi G, Lama A, Giambalvo O, Osborn J, Margiotta V, Ammatuna P. Human papillomavirus DNA in oral mucosal lesions. $\mathrm{J}$ Infect Dis 2002; 15: 833-6.

22.- Marques A E, Barra G B, de Resende Oyama C N, Guerra E N. Low rate of oropharyngeal human papillomavirus infection of women with cervical lesions and their partners: new data from Brazilian population. J Oral Pathol Med 2015; 44: 453-8.

23.- Chaturvedi A K, Graubard B I, Broutian T, Pickard R K, Tong Z Y, Xiao W, et al. NHANES 2009-2012 findings: association of sexual behaviors with higher prevalence of oral oncogenic human papillomavirus infections in U.S. men. Cancer Res 2015; 75: 2468-77.

24.- González-Losa M del R, Barrera ES, HerreraPech V, Conde-Ferráez L, Puerto-Solís M, Ayora-Talavera G. Epidemiology of oral HPV in the oral mucosa in women without signs of oral disease from Yucatan, Mexico. Braz J Microbiol 2015; 46: 301-6.

25.- Cuberos V, Pérez J, López C J, Castro F, González L V, Correa L A, et al. Molecular and serological evidence of the epidemiological association of HPV 13 with focal epithelial hyperplasia: a case-control study. J Clin Virol 2006; 37: 21-6.

26.- Antonsson A, Cornford M, Perry S, Davis M, Dunne M P, Whiteman D C. Prevalence and risk factors for oral HPV infection in young Australians. PLoS One 2014; 9: e91761.

27.- Power J, McNair R, Carr S. Absent sexual scripts: lesbian and bisexual women's knowledge, attitudes and action regarding safer sex and sexual health information. Cult Health Sex 2009; 11: 67-81.

28.- Curmi C, Peters K, Salamonson Y. Lesbians' attitudes and practices of cervical cancer screening: a qualitative study. BMC Womens Health 2014; 14: 153.

29.- Reis H L, Ferreira D C, Forattini A G, Souza P G, Curvelo J A, Passos M R. Genital and oral human papillomavirus infection in a patient from the group of women who have sex with women. Clinics (Sao Paulo) 2010; 65: 1383-5.

30.- Bailey J V, Kavanagh J, Owen C, McLean K A, Skinner C J. Lesbians and cervical screening. Br J Gen Pract 2000; 50: 481-2.

31.- Kreimer A R, Pierce Campbell C M, Lin H Y, Fulp W, Papenfuss M R, Abrahamsen M, et al. Incidence and clearance of oral human papillomavirus infection in men: the HIM cohort study. Lancet 2013; 382: 877-87.

32.- van Aar F, Mooij S H, van der Sande M A, Meijer C J, King A J, Verhagen D W, et al. Twelve-month incidence and clearance of oral HPV infection in HIV-negative and HIVinfected men who have sex with men: the H2M cohort study. BMC Infect Dis 2014; 14: 668. 